ORNL/TM--11562

DE91 000517

Fusion Energy Division

\title{
MODEL FOR PARTICLE BALANCE IN PUMPED DIVERTORS (pre-VORTEX)
}

\author{
J. T. Hogan
}

Date Published: August 1990

Prepared for the

Office of Fusion Energy

Budget Activity No. AT 05

Prepared by the

OAK RIDGE NATIONAL LABORATORY

Oak Ridge, Tennessee 37831-6285 operated by

MARTIN MARIETTA ENERGY SYSTEMS, INC

for the

U.S. DEPARTMENT OF ENERGY

under contract DE-AC05-840R21400 


\section{CONTENTS}
ABSTRACT
$\mathrm{v}$
1. INTRODUCTION _..........................................................................

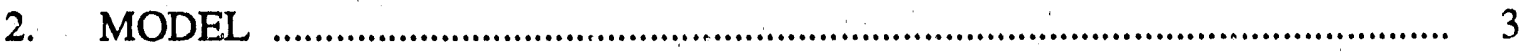
2.1 DIVERTOR CHANNEL ……....................................................... 3

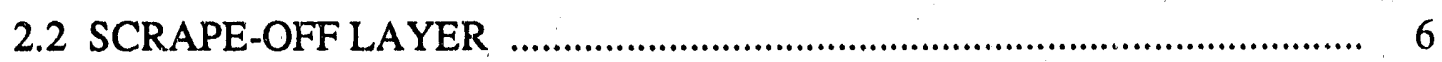

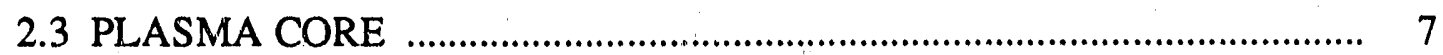
2.4 NEUTRAL BALANCE _............................................................... 8

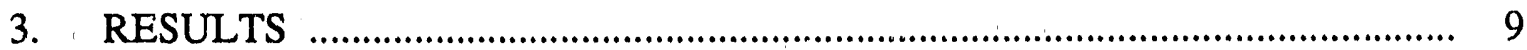

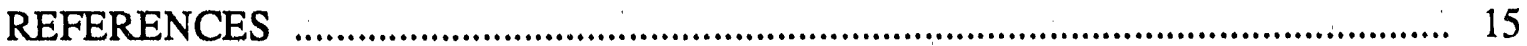




\begin{abstract}
An internally consistent model for particle transport in an open divertor geometry has been developed. Embodied in a new code, pre-VORTEX, the model couples the particle balance in the plasma core, the scrape-off layer, the open divertor channels, and the "vacuum" regions. This mutual coupling is particularly important in determining the conditions required for high recycling in the divertor. The plasma core is considered to have a relatively quiescent core region and a less well confined "edge-localized mode" (ELM) region. The scrape-off layer is modeled with one-dimensional parallel and prependicular transport. A two-point divertor channel model is used; it is similar to previous models, but with the addition of new physical processes: hydrogen charge exchange, impurity thermal charge exchange, and flux-limited parallel transport. Wall recycling data are required to describe the differing recycling properties of the wall regions and the divertor plates. Given local plasma diffusivities and wall recycling properties, the model predicts the volume-averaged density and global particle confinement time. The input data are uncertain, and a major use for the model is to permit comparison with data. The final model, VORTEX, is intended for application to the analysis of divertor confinement experiments; it is coupled to a one-and-one-halfdimensional transport code and uses detailed geometric input from equilibrium fitting codes, experimentally measured core profiles, and such parameters as can be measured in the scrape-off layer. The pre-VORTEX model is compared as a stand-alone code with typical data from the DIII-D experiment and applied to the proposed DIII-D Advanced Divertor Project.
\end{abstract}




\section{INTRODUCTION}

The divertor has been the object of study for some time. As described by the ASDEX group, the divertor creates a localized recycling vortex, in which a large particle flux (typically an order of magnitude greater than that of the core plasma efflux) is established at the divertor plate. ${ }^{1}$ The conditions for maintaining this high-recycling state are critical for the design of a practical divertor in future devices. ${ }^{2}$

Quantitative analysis of particle confinement in open divertors requires the establishment of a self-consistent, empirically based model for recycling processes. Unlike the previously analyzed case of closed divertors, the typical single-null open divertor allows the exchange of particle flux from one divertor channel directly to the other through the "private flux" region. There are many additional pathways for particle flows, and they are strongly coupled during the typical pulse length (1-30 s) of present tokamaks. (See Fig. 1.) In addition, schemes such as that envisaged for the DIII-D Advanced Divertor Project ${ }^{3}$ seek to control the density through active pumping at the outer divertor (region 7 in Fig. 1). Analysis of such effects requires a self-consistent model for the global particle flows.

The model described here incorporates a global description of the particle balance in a single-null open divertor and is intended to complement detailed modeling of neutrals using three-dimensional Monte Carlo codes and two-dimensional plasma scrape-off layer modeling. While the latter incorporate a high degree of geometric realism, the plasma codes are still under development, and coupling of the multidimensional codes is required to make a self-consistent model. This has rarely been done in the past, primarily bacause of the computational resources required. Also, significant changes are observed in the plasma particle balance that correspond to changes in the wall conciitioning method, and detailed models are not required for this problem.

This report describes the pre-VORTEX code, in which the models for the scrapeoff layer, divertor channels, and wall recycling are coupled to a simplified plasma model. This development has been rarried out as a precursor to the creation of a model in which the plasma is treated with a one-and-one-half-dimensional code, with a detailed description of geometry, plasina core transport, heating, and impurity efects.

Pre-VORTEX treats the parallel mass, momentum, and energy transport for the divertor channels. The scrape-off layer is treated with one-dimensional radial and parallel diffusivities. Neutral gas balance is calculated for the various regions accessible 
ORNL-DWG 90-2657 FED

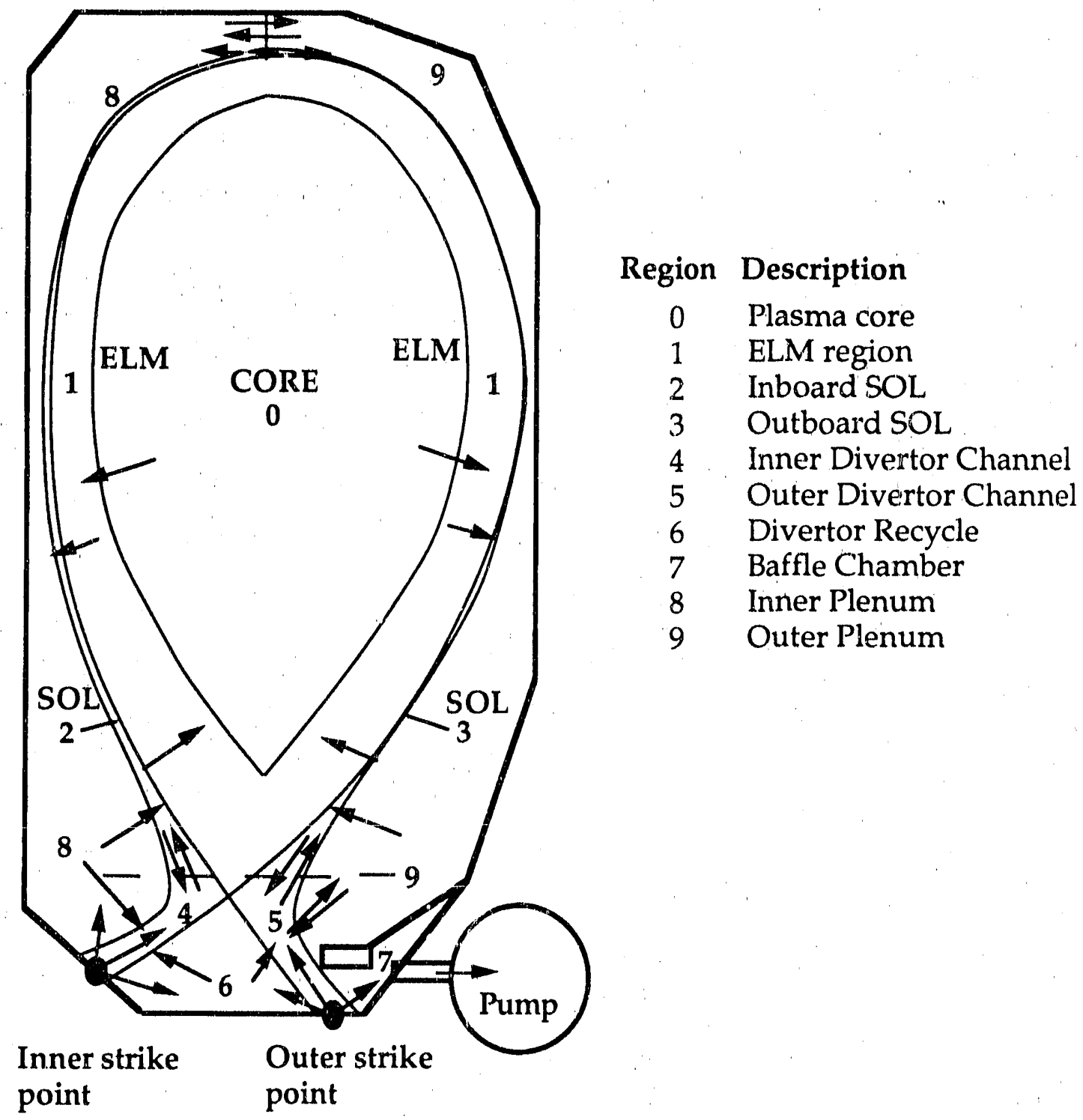

Fig. 1. Schematic view of regions described in the VORTEX code (DIII-D global divertor model). 
for neutrals inside the vessel, and exchange with the wall is treated. The core plasma is divided into a central region and an edge-localized mode (ELM) region, and the particle efflux is expressed in terms of the conventional diffusivity and the ELM frequency and amplitude. Section 2 describes the semi-analytical model, and Sect. 3 presents results.

\section{MODEL}

The most important requirement for the model is to delineate the conditions required for the establishment of a low-temperature, high-density, high-recycling divertor. Thus, the divertor channel model is described in Sect. 2.1. Then we describe the scrape-off layer equations, which couple the inner and outer channels of the divertor, and the plasma equations for both the core and the ELM region and the wall model.

\subsection{DIVERTOR CHANNEL}

The divertor channel model is based on earlier work on simplified divertor models. ${ }^{4}$ The mass balance equation for the inner divertor channel (region 4 in Fig. 1) is the simplest, since there is no external pump to be applied to this segment. The axial particle balance is as follows:

mass,

$$
\frac{1}{b(l)} \frac{\partial}{\partial l}[b(l) n v]=S_{0}+\Sigma_{\text {plasma }}
$$

momentum,

$$
\frac{1}{b(l)} \cdot \frac{\partial}{\partial l}\left[b(l) n\left(v^{2}+v_{s}^{2}\right)\right]=-\frac{n v}{\tau_{\mathrm{cx}}}
$$

energy,

$$
-\frac{1}{b(l)} \frac{\partial}{\partial l}\left[b(l) K_{0} T^{5 / 2} \frac{\partial T}{\partial l}\right]=-\alpha S_{0}-\frac{n T}{\tau_{\alpha}}
$$

Here $n, T$, and $v$ are the plasma density, temperature, and parallel flow speed; $S_{0}$ is the production rate of cold ions; $\Sigma_{\text {plasma }}$ is the efflux from the plasma into the scrape-off layer; $\alpha$ is the energy loss per ion-electron pair created (which can be enhanced to model 
low- $Z$ impurity radiation); and $\tau_{\mathrm{cx}}$ is the charge-exchange lifetime. The flux tube is assumed to have a width $b(l)$, taken from magnetic analysis. The poloidal sound speed is

$$
v_{s}^{2} \equiv \frac{B_{\text {pol }}^{2}}{B_{T}^{2}} \sqrt{\frac{2 k T}{M}} .
$$

The global model requires the net fluxes; thus, integrating from the divertor throat to the plate,

$$
\begin{aligned}
\Phi_{\text {plate }}=\Phi_{\text {throat }} & +\frac{F_{\text {plate }}(1-\delta)}{\lambda_{\|}} \int_{\text {throat }}^{\text {plate }} d l b(l) \exp \left(\frac{l-L}{\lambda}\right) \\
& +\frac{1}{4} \sum_{i=1}^{N} \Gamma_{i}\left(L_{\perp}^{i} / L\right)\left[1-\int_{\text {throat }}^{\text {plate }} d l \exp \frac{-b(l)}{\lambda_{\perp}}\right]
\end{aligned}
$$

where $\Gamma_{i}$ is the neutral influx for the channel region from adjacent zones ( 6 and 8 , for the inner channel), $L_{\perp}$ is the poloidal extent of the area through which neutrals enter the divertor channel (which has poloidal length $L$ ), $N$ is the total number of regions that contribute a neutral flux to this divertor channel, $\Phi_{\text {plate }}$ and $\Phi_{\text {throat }}$ are the particle fluxes (particles per unit area per second) at the divertor plate and throat, and $\delta$ is the fraction of ions striking the plate that escape from the reflux stream, either through absorption into the divertor plate, escape to the pump, or transit to regions 6 or 8 in Fig. 1. This fraction is expressed, for channel 4 , as

$$
\delta_{4}=\left(1-R_{4}\right)\left\{1-\left(\eta_{46}+\eta_{48}\right)\left[\exp \left(-b_{4} / \Lambda_{4}\right)\right]\left[1-\exp \left(-L_{4} / \lambda_{4}\right)\right]\right\},
$$

where $R_{4}$ is the reflection probability and $\eta_{46}$ and $\eta_{48}$ are the probabilities of escape into the central vacuum area (6) and the inner vacuum area (8), respectively.

The corresponding quantity for the outer divertor channel is

$$
\delta_{5}=\left(1-R_{5}\right)\left\{1-\left(\eta_{56}+\eta_{59}+\eta_{57}\right)\left[\exp \left(-b_{5} / \Lambda_{5}\right)\right]\left[1-\exp \left(-L_{5} / \lambda_{5}\right)\right]\right\} \text {. }
$$

The perpendicular $(\Lambda)$ and parallel $(\lambda)$ mean free paths for ionization are computed from the plate and throat temperatures and densities,

$$
\Lambda=\frac{v_{\text {th,plate }}}{n_{\text {plate }}\langle\sigma \nu\rangle_{\text {ion,plate }}}, \lambda=\frac{v_{\text {th,plate }}}{n_{\text {plate }}\langle\sigma \nu\rangle_{\text {ion,throat }}} .
$$


Integration of these equations leads to solutions for the plate and throat temperatures and densities required for a steady state.

The flux amplification factor is

$$
A=\frac{1}{D}\left\{1+\frac{G}{\Phi_{\text {throat }}}\left[1-\frac{\Lambda}{\delta b} \exp (-\{\delta\} b / \Lambda)\right]\right\},
$$

where

$$
D=1-(1-\delta)\left[1-\varepsilon \exp (-L / \lambda)-(1-\varepsilon) \frac{\lambda}{L}\left(1-\exp \frac{-L}{\lambda}\right)\right]
$$

and

$$
G=2 \pi R L \sum_{i=1}^{N}\left(\frac{\Gamma_{I}^{P}}{4}\right)\left(\frac{L_{I}^{P}}{L}\right)
$$

The expression for the plate temperature is

$$
T_{\text {plate }}=\frac{Q_{\text {throat }}}{\gamma A \Phi_{\text {throat }}}-\frac{\alpha}{\gamma} \frac{A-1}{A}
$$

where $Q_{\text {throat }}$ is the power per unit area entering the throat, $\gamma$ is the secondary electron emission coefficient, $R$ is the average major radius for this divertor channel, and $\delta b$ is the variation of the divertor channel width. In this pre-VORTEX model, the channel width is taken as $b(l)=b_{\text {throat }}+\delta b\left(l-l_{\text {throat }}\right) / L$. The throat temperature is

$$
T_{\text {throat }}=\left\{\frac{T_{\text {plate }}^{7 / 2}}{\varepsilon}+\frac{7}{2 K_{0}} \frac{L \Phi_{\text {throat }}}{2 \pi R b_{\text {throat }}}\left[\frac{Q_{\text {throat }}}{\Phi_{\text {throat }}}-\alpha(H)\right]\right\},
$$

where $H=\left[A(1-\delta) T_{1}+\left(G / \Phi_{\text {throat }}\right) T_{2}\right]$, with

$$
\begin{gathered}
T_{1}=\frac{\lambda}{L}[1-\exp (-L / \lambda)]\left[\varepsilon-\frac{\lambda}{L}(1-\varepsilon)\right]-\varepsilon \exp (-L / \lambda) \\
+(1-\varepsilon) \frac{\lambda}{L}\left[\frac{\lambda}{L} \exp (-L / \lambda)+\left(1-\frac{L}{\Lambda}\right)\right]
\end{gathered}
$$


and

$$
T_{2}=\frac{1}{2}-\frac{\lambda}{b_{\text {plate }}}(1-\varepsilon)^{-1} \exp \left(-b_{\imath} / L\right)\left\{1-\frac{\lambda}{\delta b} \frac{1-\exp \left[-b_{p}(1-\varepsilon) / \lambda\right]}{1-\varepsilon}\right\}
$$

For $v_{\text {throat we use }}$

$$
v_{\text {throat }}=c_{s, \text { throat }}\left[\frac{\Phi_{\text {plate }}}{\Phi_{\text {throat }}} \sqrt{\frac{T_{\text {plate }}}{T_{\text {throat }}}}-\sqrt{\left(\frac{\Phi_{\text {plate }}}{\Phi_{\text {throat }}}\right)^{2} \frac{T_{\text {plate }}}{T_{\text {throat }}}-1}\right] .
$$

\subsection{SCRAPE-OFF LAYER}

The mass, momientum, and energy balance equations are used to connect the inner and outer divertor channels through the scrape-off layer. No explicit assumption is made with respect to the existence of a "watershed," or symmetry, point. Instead, the inner and outer scrape-off layer solutions are matched by requiring continuity. This approach is suggested both by the observed asymmetry in particle and energy fluxes in divertor experiments and by the plan to bias the divertor plates in the DIII-D Advanced Divertor Project to influence scrape-off layer properties.

The resulting equations are

$$
\Phi_{5}-\Phi_{4}=\Gamma_{82}+\Gamma_{93}+\Sigma_{p} f_{3}+\Sigma_{p}\left(1-f_{3}\right)
$$

and

$$
Q_{5}-Q_{4}=-\alpha\left(\Gamma_{82}+\Gamma_{93}\right)+P_{\text {in }} / 2 \pi R b
$$

where $\Phi_{4}$ and $Q_{4}$ are negative (counter-clockwise) for a symmetric case. inese equations thus express the requirement that the total particle flux $\left(\Sigma_{\mathrm{p}}\right)$ and heat flux $\left(P_{\text {in }} / 2 \pi R b\right)$, modified for ionization of the neutral influxes $\Gamma_{82}$ and $\Gamma_{93}$, must be transported in steady state.

The momentum and energy balance equations couple the inner and outer throat regions:

$$
\frac{2 \chi_{\|}}{7}\left(T_{4}^{7 / 2}-T_{5}^{7 / 2}\right)=L_{3} Q_{5}+L_{2} Q_{4}+\frac{\alpha}{2}\left(L_{3} \Gamma_{93}-L_{2} \Gamma_{82}\right)-\frac{1}{2} \frac{P_{\text {in }}}{2 \pi r b}\left[L_{3} f_{3}-L_{2}\left(1-f_{3}\right)\right]
$$


and

$$
\frac{F_{4}^{2}}{n_{4}}+\frac{B_{\mathrm{pol}}^{2}}{B_{T}^{2}} \frac{2 T_{4}}{M}=\frac{F_{5}^{2}}{n_{5}}+\frac{B_{\mathrm{pol}}^{2}}{B_{T}^{2}} \frac{2 T_{5}}{M}
$$

The scrape-off layer density is determined by a balance between parallel and perpendicular particle fiuxes,

$$
-D_{\perp} d^{2} N_{\mathrm{e}} / d x^{2}=S_{\text {plasma }}+S_{\text {ioniz }}-N_{\mathrm{e}} / \tau_{\|}
$$

where $S_{\text {plasma }}$ is the efflux from the plasma into the scrape-off layer, $S_{\text {ioniz }}$ is the particle source due to neutrals from the wall or divertor impinging on the scrape-off layer, $D_{\perp}$ is the cross-field diffusivity, and $N_{\mathrm{e}} / \tau_{\|}$is the parallel flow loss of scrape-off layer particles to the divertor throats. This gives the solution

$$
N_{\text {sep }}=\left(\Gamma_{0}+\Sigma_{0} a_{\mathrm{ELM}} / a\right)\left(\tau_{\|} D_{\perp}\right)^{1 / 2} / D_{\mathrm{ELM}}
$$

where $N_{\text {sep }}$ is the plasma density at the separatrix (which is determined from the core solution) and $a_{E L M}$ is the radius outside which ELM behavior is assumed to dominate transport ( $a_{E L M}$ enters because the effective density yradient at the edge depends on it).

\subsection{PLASMA CORE}

The plasma core model is

$$
\begin{array}{ll}
-D_{\text {core }} d^{2} N_{\mathrm{e}} / d r^{2}=\Sigma_{\text {fuel }}, & 0<r<a_{\mathrm{ELM}} \\
-D_{\mathrm{ELM}} d^{2} N_{\mathrm{e}} / d r^{2}=N_{\mathrm{e}} N_{0} S_{\text {ioniz }}, & a_{\mathrm{ELM}}<r<a \\
V_{0} d N_{0} / d r=-N_{\mathrm{e}} N_{0} S_{\text {ioniz }}, & a_{\mathrm{ELM}}<r<a
\end{array}
$$

where $D_{\text {ELM }}$ is a time-averaged diffusion coefficient based on the ELM frequency and amplitude, $N_{0}$ is the neutral density in the core, $S_{\text {ioniz }}$ is the rate coefficient for electron impact ionization $(\langle\sigma v\rangle), V_{0}$ is the neutral velocity in the core, and all core ionization is assumed to be localized in the ELM region.

The solution for the plasma model relates the volume-average density (hence the particle confinement time) to the separatrix density: 


$$
\begin{aligned}
\left\langle N_{\mathrm{e}}\right\rangle=N_{\text {sep }} & +\left(\Gamma_{0} V_{0} / S_{\text {ioniz }} D_{\text {ELM }}\right) / N_{\text {sep }} \\
& +\left(a_{\text {ELM }} \Sigma_{\text {fuel }} / D_{\text {core }}\right)\left\{D_{\text {core }} / D_{\text {ELM }}\left[1-\left(a_{\text {ELM }} / a\right)^{2}\right] / 2+\left(a_{\text {ELM }} / a\right)^{2} / 3\right\}
\end{aligned}
$$

and

$$
\tau_{\mathrm{p}}=\left\langle N_{\mathrm{e}}\right\rangle /\left(\Sigma_{\text {fuel }}+\Sigma_{\text {ioniz }}\right)
$$

\subsection{NEUTRAL BALANCE}

To form a self-consistent model the neutral balance is calculated for regions 6-9 in Fig. 1. Thus in region 6 (private flux),

$$
\begin{aligned}
\dot{N}_{6}=0 & =\Gamma_{4} \eta_{4,6}+\Gamma_{5} \eta_{5,6}+\frac{1}{4}\left(1-\eta_{9,5}\right) n_{9} v_{9} A_{9,5} \\
& +\frac{1}{4}\left(1-\eta_{8,4}\right) n_{8} \nu_{8} A_{8,4}-\frac{1}{4} n_{6} v_{6} A_{6,4} \\
& -\frac{1}{4} n_{6} v_{6} A_{6,5}+\frac{1}{4}\left(1-\eta_{5,7}\right) n_{7} \nu_{7} A_{7,5}
\end{aligned}
$$

in region 7 (pumping chamber),

$$
\begin{aligned}
\dot{N}_{7}= & =\Gamma_{5} \eta_{7}^{\text {plate }}+\frac{1}{4}\left(1-\eta_{6,9}\right)\left(1-\eta_{6,5}\right) n_{6} \nu_{6} A_{6,5} \\
& -S n_{7}\left(k T_{7}\right)-\frac{1}{4} n_{7} \nu_{7} A_{7,5}
\end{aligned}
$$

in region 8 (inner plenum),

$$
\begin{aligned}
\dot{N}_{8}=0 & =\Gamma_{4} \eta_{8}^{\text {plate }}+\frac{1}{4}\left(1-\eta_{6,4}\right) n_{6} v_{6} A_{6,4} \\
& -\frac{1}{4} n_{8} v_{8} A_{8,4}-\frac{1}{4} n_{8} v_{8} A_{8,2} ;
\end{aligned}
$$

and in region 9 (outer plenum),

$$
\begin{aligned}
\dot{N}_{9}= & =\Gamma_{5} \eta_{9}^{\text {plate }}+\frac{1}{4}\left(1-\eta_{6,5}\right) \eta_{6,9} n_{6} v_{6} A_{6,5} \\
& -\frac{1}{4} n_{9} v_{9} A_{9,5}-\frac{1}{4} n_{9} v_{9} A_{9,3} .
\end{aligned}
$$




\section{RESULTS}

The pre-VORTEX model has been applied to a DIII-D divertor case to predict the range of values to be expected when divertor pumping is applied, as is proposed for the DIII-D Advanced Divertor Project. Using a data set originally compiled for DIII-D,5 we consider the effects of applying up to $50,000 \mathrm{~L} \cdot \mathrm{s}$ of pumping to a plasma with $5 \mathrm{MW}$ of injected power and 50 to $250 \mathrm{~A}$ of central fueling. The data required for this case are shown in Table 1.

The wall reflection coefficients $R_{6}, R_{8}$ and $R_{9}$ are taken as 0.98 , the wall reflection coefficient in the pump region $R_{7}$ is 0.99 , and the divertor reflection coefficients $R_{4}$ and $R_{5}$ are also assumed to be 0.98 . The gap at the pump inlet is $3.5 \mathrm{~cm}$, and room-temperature particles are assumed to be recycled there.

The plasma diffusivities $D_{\text {core }}$ and $D_{\text {ELM }}=5 \times 10^{3} \mathrm{~cm}^{-2} / \mathrm{s} ; x_{\mathrm{ELM}}=1.0$, and $D_{\perp}=$ $5 \times 10^{3} \mathrm{~cm}^{-2} / \mathrm{s}$. The plasma efflux is assumed to be predominantly distributed to the outside $\left(f_{3}=0.7\right)$.

With this reference data set, the variation of plasma core, SOL, divertor, and pump parameters is calculated for the nominal range of pumping speeds available for DIII-D: $S=0-60,000 \mathrm{~L} / \mathrm{s}$. Results for a sample case are given in Table 2 . The results of the analysis for variation in pumping speed are shown in Figs. 2-5.

Figure 2(a) shows the variation in the outer divertor flux amplification factor with pumping speed. The amplification factor decreases from 10 to $\sim 5$ without significant core fueling but is maintained in the high-recyclirig condtion $(A \sim 10)$ with core fueling of 150 to $250 \mathrm{~A}$. The core density decreases by up to $50 \%$ without core

Table 1. Data required for applying pre-VORTEX

\begin{tabular}{lcccc}
\hline & \multicolumn{2}{c}{ Divertor } & \multicolumn{2}{c}{ Scrape-off layer } \\
\cline { 2 - 5 } & Inner & Outer & Inner & Outer \\
\hline Region (see Fig. 1) & 4 & 5 & 2 & 3 \\
Channel width $b, \mathrm{~cm}$ & 4.0 & 2.0 & 1.0 & 1.0 \\
Poloidal length $L, \mathrm{~cm}$ & 30 & 40 & 200 & 260 \\
$B_{T} / B_{\text {pol }}$ & 33 & 33 & 33 & 33 \\
Reflection coefficient & & & & \\
$R(\mathrm{~cm})$ & 1.5 & 1.9 & 1.5 & 1.9 \\
$\kappa_{\|}$ & 0.9 & 0.9 & 0.9 & 0.9 \\
\hline
\end{tabular}


Table 2. Results for sample case

Power $=6.0 \mathrm{MW}$, pumping speed $=6 \times 10^{4} \mathrm{~L} / \mathrm{s}$, average core density $=0.49 \times 10^{20} \mathrm{~m}^{-3}, \tau_{\mathrm{p}}=0.238 \mathrm{~s}$

Region
$Q, \mathrm{MW}$
$I, \mathrm{~A}$
$N_{\text {plate }}, 10^{20} \mathrm{~m}^{-3}$
$N_{\text {throai }}, 10^{20} \mathrm{~m}^{-3}$
$T_{\text {plate }}, \mathrm{eV}$
$T_{\text {throat }}, \mathrm{eV}$
$A$ Neutral pressure, torr
Paiticle flux, A
To plate
From plate
To private
From plenum
To scrape-off layer

Particle flux, A

From plenum

From plate

Plasma efflux

\section{Divertor}

Quter

5

4.23

777.0

0.45

0.81

37.4

41.2

7.1

0.0003

469.4

4532.4

111.8

16.5

777.3
Inner

4

$-1.77$

$-296.0$

0.01

0.01

366

366

1.1

0.0003

313.9

16.1

2.0

0.27

296.3

\section{Scrape-off layer}

Quter

81.1

7.01

689.2
Inner

0.45

0.52

295.4

\section{Plasma core}

Particle flux , A

Fuel

Reflux, region 4 260.4

Reflux, region 5

Influx, region 8

Influx, region 9

Efflux, region 2 
Table 2 (continued)

\begin{tabular}{|c|c|c|}
\hline \multicolumn{3}{|c|}{ Pump } \\
\hline Neutral pressure, torr & & 0.362 \\
\hline \multicolumn{3}{|l|}{ Particle flux, A } \\
\hline To plate & & 396 \\
\hline To private & & 27.7 \\
\hline Pumped & & 226.9 \\
\hline Back, region 5 & & 35.3 \\
\hline Back, region 6 & & 99.9 \\
\hline \multirow[t]{2}{*}{ Wall pumped } & & 61.6 \\
\hline & Private flux & \\
\hline \multirow[t]{2}{*}{ Neutral pressure, torr } & & 0.002 \\
\hline & Global efficiencies & \\
\hline Fraction diverted, $\%$ & & 6.9 \\
\hline Fraction trapped, \% & & 58.4 \\
\hline Exhaust fraction, \% & & 23.1 \\
\hline
\end{tabular}

fueling [Fig. 2(b)] and is relatively constant with 150 to $130 \mathrm{~A}$ of core fueling. Thus, the density profile should be strongly modified by pumping.

The divertor plasma parameters are shown in Fig. 3. The plate temperature rises to $\sim 50 \mathrm{eV}$ without core fueling (as the amplification factor drops to 5) but is in the 10- to $20-\mathrm{eV}$ range for core fueling rates of 150 to $250 \mathrm{~A}$. Likewise, the divertor electron density can be kept at a high value (to reduce backflow of recycled neutrals) for the higher core fueling rates.

The core plasma behavior is shown in Fig. 4. The average core electron density drops significantly with pumping rates of 30,000 to $50,000 \mathrm{~L} / \mathrm{s}$ with low core fueling, but can be maintained with 150 to $250 \mathrm{~A}$. The particle confinement time is calculated to improve with high pumping and core fueling.

Finally, the overall exhaust efficiency of the pumping is shown in Fig. 5. The efficiency rises with an increase in pumping speed and core fueling, reaching values around $25 \%$. 

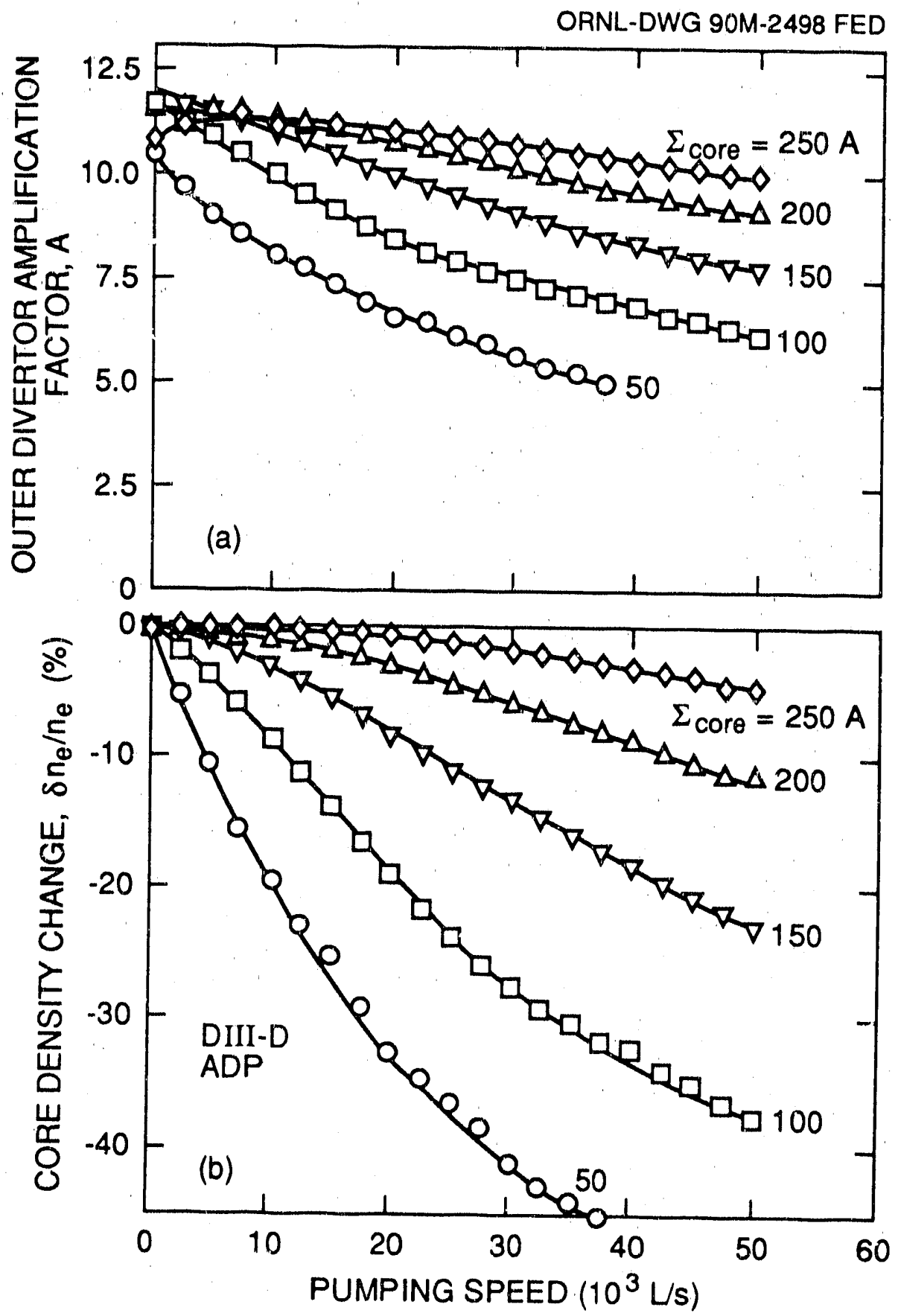

Fig. 2. (a) Variation in the outer divertor flux amplification factor with pumping speed. (b) Change in core density with pumping speed. 

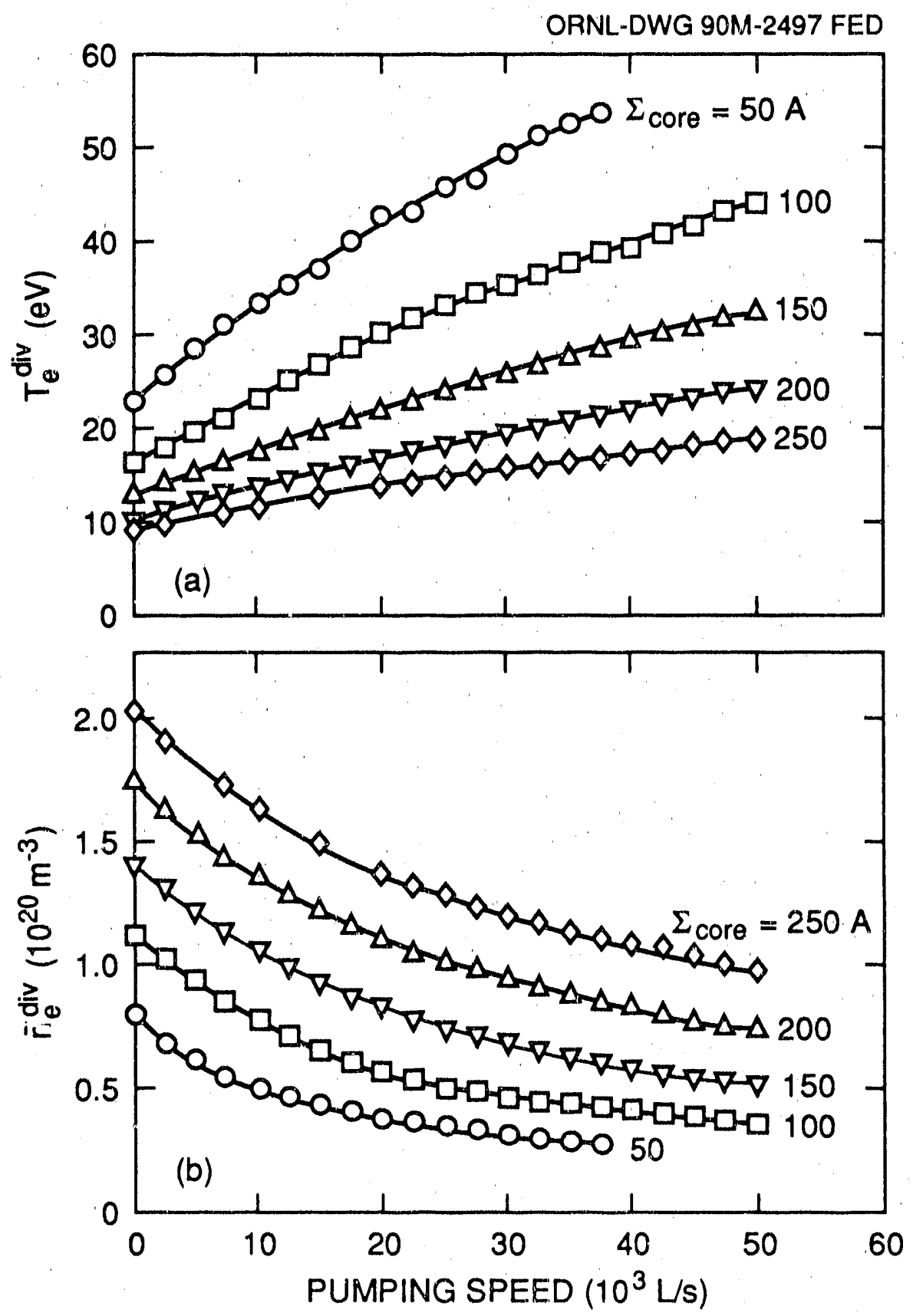

Fig. 3. (a) Temperature and (b) density of the divertor plasma as functions of pumping speed. 
ORNL-DWG 9OM-2658 FED

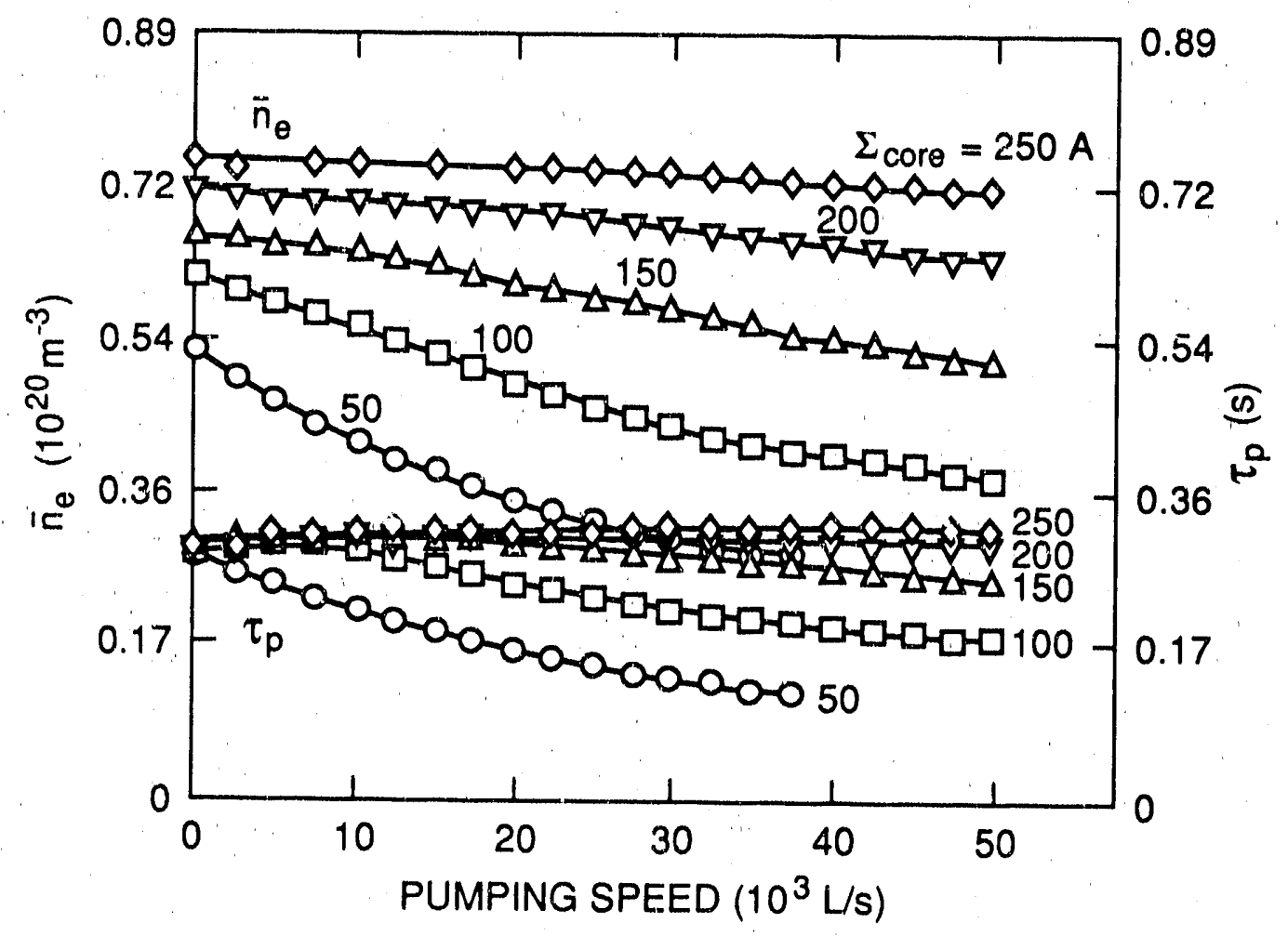

Fig. 4. Core plasma behavior.

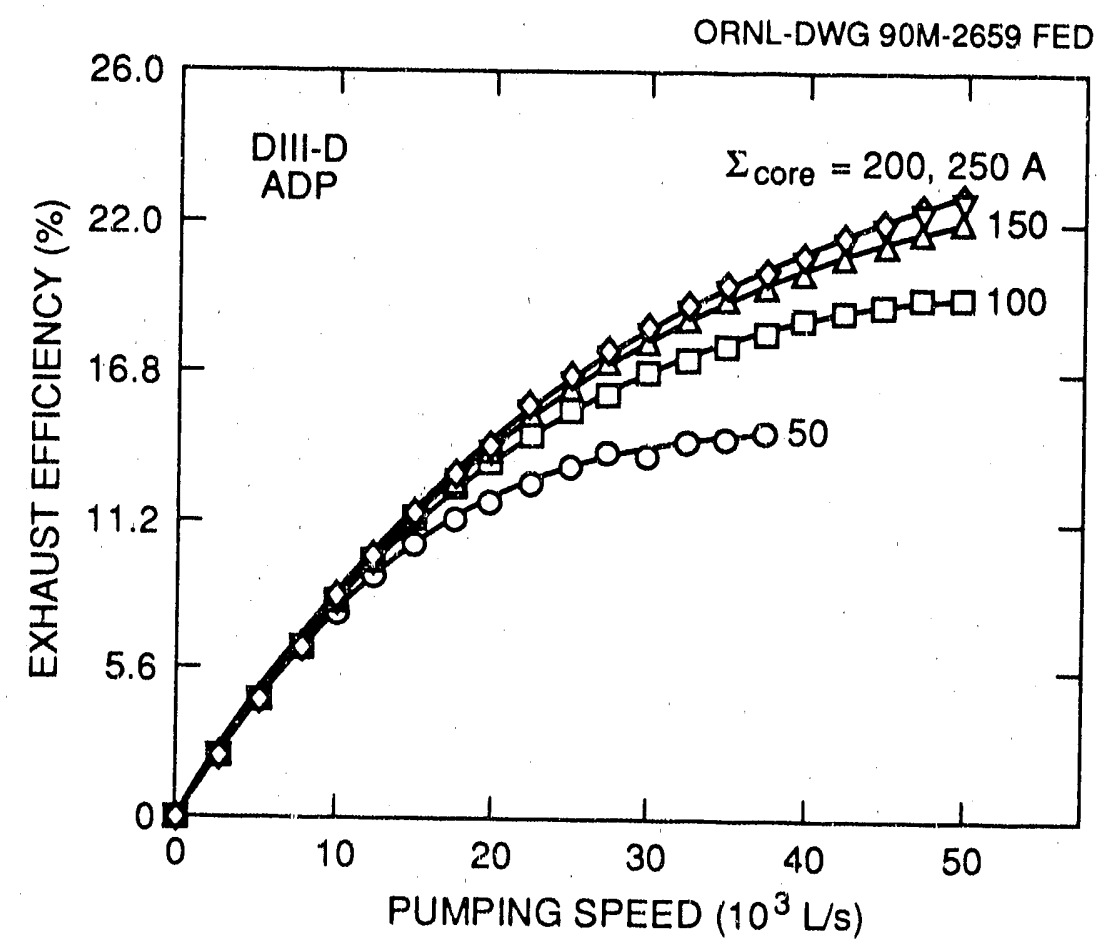

Fig. 5. Exhaust efficiency as a function of pumping speed. 


\section{REFERENCES}

1. F. Wagner and K. Lackner, in Physics of Plasma-Wall Interactions in Controlled Fusion, ed. D. Post and R. Behrisch, Plenum, New York, 1986.

2. ITER Conceptual Design Interim Report, International Atomic Energy Agency, Vienna, October 1989.

3. A. Mahdavi et al., The DIII-D Collaborative Advanced Divertor Program, GA-A19547, General Atomics, San Diego, Calif., February 1989.

4. S. Saito et al., Nucl. Fusion 25, 828 (1985); D. E. Post, W. Langer, and M. Petravic, J. Nucl. Mater. 121, 171 (1984).

5. M. E. Rensink et al., "Particle Confinement Times for Divertor Discharges in DIII-D," Bull. Am. Phys. Soc. 33, 1965 (1988). 

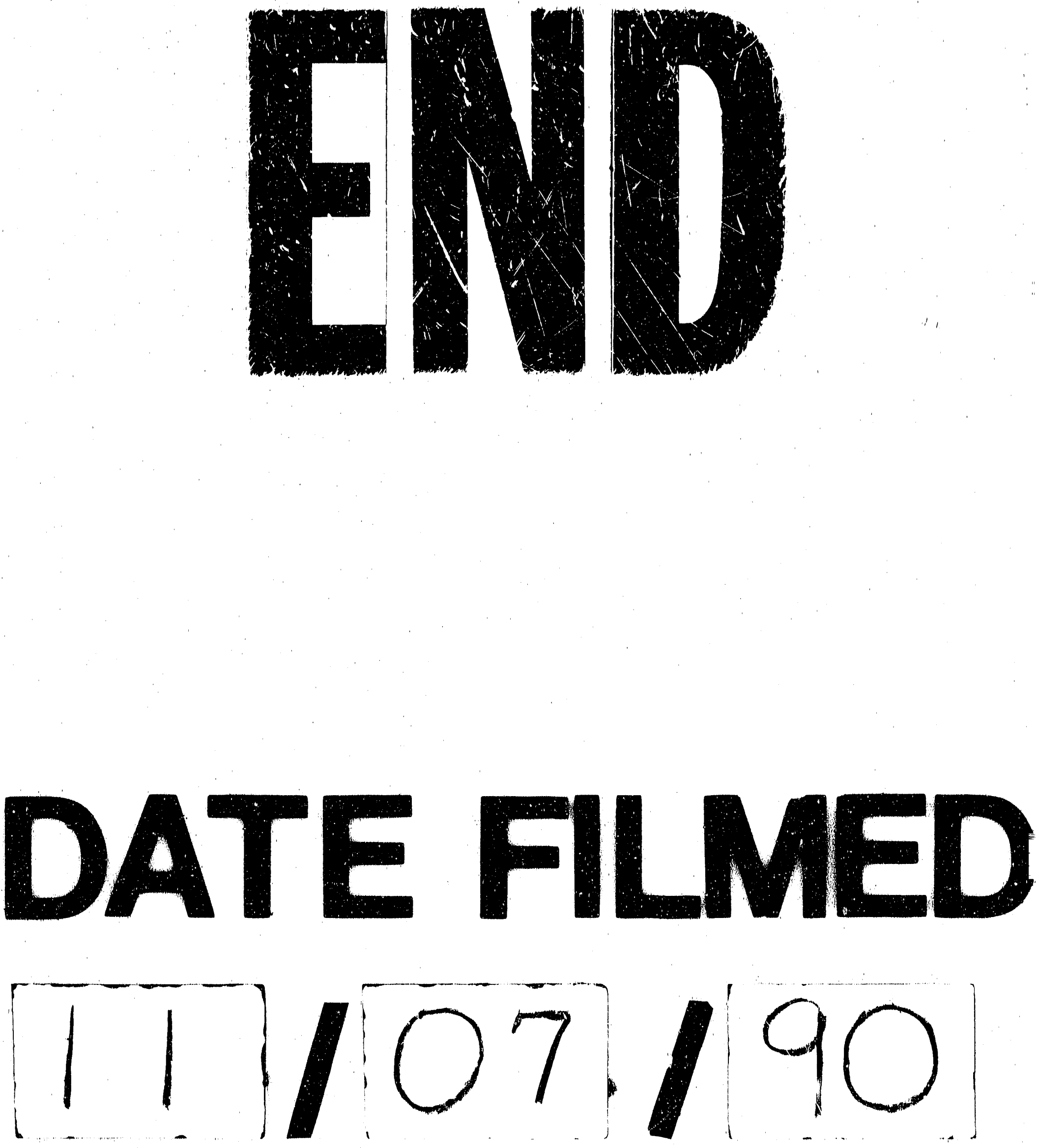
